\title{
Cooperative MIMO for Adaptive Physical Layer Security in WBAN
}

\author{
Amel Arfaoui**, Ali Kribecheł, Sidi Mohammed Senouci \\ ${ }^{\ddagger}$ DRIVE EA1859, Univ. Bourgogne Franche Comté, France \\ *Digital Security Unit, SupCom University of Carthage, Tunisia \\ \{amel.arfaoui, Sidi-Mohammed.Senouci, Ali.Kribeche01\}@u-bourgogne.fr
}

\begin{abstract}
Internet of Things (IoT) is becoming an emerging paradigm to provide pervasive connectivity where "anything" can be connected "anywhere" at "anytime" via massive deployment of physical objects like sensors, controllers, and actuators. However, the open nature of wireless communications and the energy constraint of the IoT devices impose strong security concerns. In this context, traditional cryptographic techniques may not be suitable in such a resource-constrained network. To address this problem, an effective security solution that ensures a trade-off between security effectiveness and energy efficiency is required. In this paper, we exploit cooperative transmission between sensor nodes in IoT for e-Health application, as a promising technique to enhance the physical layer security of wireless communications in terms of secrecy capacity while considering the resource-impoverished devices. Specifically, we propose a dynamic and cooperative virtual multiple-input and multiple-output (MIMO) configuration approach based on game theory to preserve the confidentiality of the transmitted messages with high energy savings. For this purpose, we model the physical layer security cooperation problem as a non-transferable coalition formation game. The set of cooperative devices form a virtual dynamically-configured MIMO network that is able to securely and efficiently transmit data to the destination. Simulation results show that the proposed game-based virtual MIMO configuration approach can improve the average secrecy capacity per device as well as the network lifetime compared to non-cooperative transmission.
\end{abstract}

Keywords-, IoT, Virtual MIMO, Physical Layer Security, Game theory, Coalitional Game.

\section{INTRODUCTION}

Security and privacy preservation are fundamental requirements for the Internet of Things (IoT) applications. Particularly, the open and broadcast nature of wireless communications in IoT make the transmitted data (uplink transmission from sensors to controllers, downlink communication from controllers to actuators, etc.) prone to several security threats such as eavesdropping attacks. The conventional way to address security issues is the adoption of cryptographic mechanisms implemented in upper layers of the network protocol stack. Nevertheless, most of these methods face the problem of secret key distribution and management as well as high complexity [1]. Thus, it is a major challenge to implement security solutions in IoT systems while considering the resource-constrained IoT devices.

Unlike encryption-based security, physical layer security [2] exploits the inherent randomness of the wireless channel such as fading, noise, interference, etc. As an alternative or complement to complex cryptographic techniques, physical layer security which does not require pre-shared secret keys has gained huge attention from the research community as an appealing solution to secure communications in IoT [3]. One key advantage of the physical layer security is its capacity to provide information-theoretic security regardless of eavesdropper's computing capability. When dealing with physical layer security, the reliability of a wireless channel is quantified by the secrecy capacity which is defined as the maximal rate of secret information transmitted to the destination in the presence of multiple eavesdroppers.

Multiple-input multiple-output (MIMO) technique has become an emerging radio-communication technology due to its capability of having spatial degrees of freedom and diversity gains. It provides simultaneous transmission for multiple spatial streams through multiple antennas. It is considered as an efficient and promising tool for improving the secrecy capacity and reliability of wireless communication systems [4] [5]. For instance, in [6], a transmit antenna selection (TAS) scheme was proposed to improve the secrecy capacity for MIMO wiretap channels, with different receiver combining models. In [7], the authors analyzed the impact of antenna correlation on the security performance of wiretap channels with multiple antennas. Later, a multitude of sophisticated signal processing techniques have been proposed to exploit the extra degrees of freedom of MIMO for physical layer security. In [8] [9], a security-oriented beamforming approach is used to improve the secrecy capacity. In such a model, the transmitter determines the transmission signal dedicated to the destination to elevate the secrecy gains.

Game theory is a decision-making process that provides an effective tool to model the interactions between a network of users. It is recently used to deal with wireless security. In [10], a multi-hop topology formation game was formulated to improve the physical layer security while jointly optimizing the physical-layer secrecy outage probability and the end-to-end delay. Cooperative game theory deals with situations where agents can cooperate to create value [11]. The main branch of cooperative games is the coalitional game, which describes the formation of cooperative groups of nodes to enhance network performance. Coalitional game with non-transferable utility has been widely exploited to analyze network security and privacy [12]. A coalitional game is called a non-transferable utility (NTU) game, if the players have preference over coalitions and the gains cannot be divided arbitrarily between them. 
Even though cooperative transmission and MIMO techniques have received a great deal of attention due to their distinct characteristics, none of the above works has exploited their effectiveness to adaptively improve the secrecy performance in IoT. In addition, they do not consider resourceconstrained environments where the residual energy of nodes should be incorporated into the selection of the cooperative nodes in order to ensure a tradeoff between the secrecy capacity and energy efficiency. Motivated by the above discussion, we exploit the reliability of the MIMO technique to design a virtual MIMO configuration approach for massive data transmission in the IoT for e-Health application. The main idea of this work is to enable collaborative transmission between the sensor nodes in a dynamic WBAN system in order to improve the link performance and the secrecy capacity of the medical devices while considering their capabilities. For this purpose, we propose a coalition formation game for cooperative transmission among medical devices. The transmitters cooperate to null the signal at the eavesdropper, thus, enhancing the secrecy capacity. Specifically, the cooperating single antenna nodes will form a virtual MIMO communication system known as coalitions. The novelties of our proposed model consist of:

- A dynamic and adaptive virtual MIMO configuration approach that is designed to determine the most appropriate virtual MIMO configuration that ensures an optimal tradeoff between the security effectiveness (secrecy capacity) and energy efficiency,

- A coalitional game-theoretic approach to model the cooperative transmission and study the interactions between medical devices that seek to maximize their secrecy capacity through cooperation in the presence of multiple eavesdroppers,

- A distributed coalition formation algorithm where each sensor node decides to join or break a coalition for maximizing its secrecy capacity while considering its residual energy,

- Simulation results prove that the cooperative transmission through virtual MIMO outperforms the non-cooperative transmission in terms of energy consumption as well as secrecy capacity.

The remainder of the paper is organized as follows. In Section II, we present the system model. In Section III, we formulate the physical layer security in WBAN as a coalition game. Numerical simulation results are presented and analyzed in Section IV. Finally, the conclusion is drawn in Section V.

\section{SYSTEM MODEL}

In this section, we present the uplink channel model of the considered virtual MIMO system for intra-WBAN. Then, we introduce the energy consumption and power constraints model incorporated in cooperative decision-making.

\section{A. Channel Model}

We consider secure uplink transmission in WBAN where $N$ on-body sensor nodes transmit confidential information to a common controller node (IoT gateway) according to their allocated time-slot as specified by the TDMA schedule. Note that if we use TDMA transmission for multiple access, the proposed model can be extended to accommodate other schemes such as FDMA or CDMA/CA. The controller node is supposed to be equipped with multiple antennas $N_{r}$ in order to implement the MIMO transmission, while sensor nodes have a single antenna. We assume that an eavesdropper with multiantenna $N_{e}$ is present in the vicinity, to overhear the confidential messages on the uplink transmission. We denote $\mathcal{N}=\{1 \ldots N\}$ as the set of all $N$ body sensor nodes in the network, and $S \subseteq \mathcal{N}$ as a coalition that consists of $|S|$ cooperating sensor nodes $(|\cdot|$ is the set cardinality). The sensor nodes forming each coalition $S$ will subsequently perform a TDMA transmission, which is one coalition per time slot. During every slot, the coalition acts as a single entity that transmits the data of the sensor node that owns the slot. The medical devices can cooperate, using either decode-and-forward (DF) or amplify-and-forward (AF). The transmitted signals from cooperative sensor nodes are combined before arriving at the receiver. Space-Time Block Coding (STBC) can be executed at both transmitters and receivers to separate the received signals and provide a diversity gain. The corresponding system model is illustrated in Figure 1 .

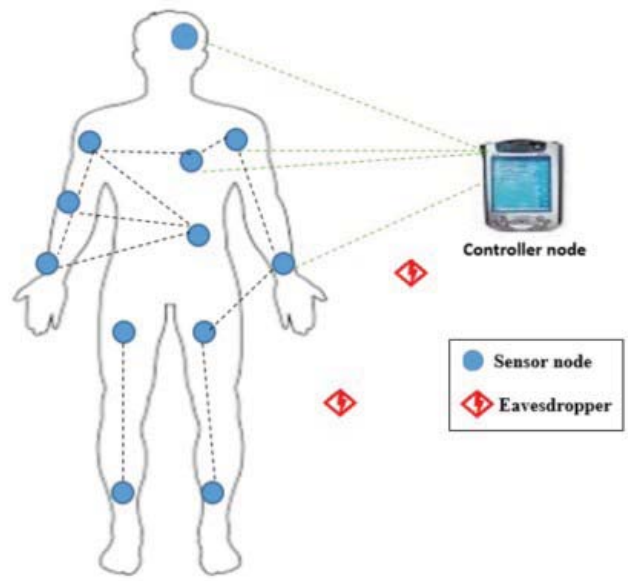

Figure 1. System model

The communication between the controller node and the coalition $S$ of transmitters is modeled according to the Gaussian MIMO wiretap channel with a legitimate receiver and an eavesdropper. They are given as follows [13]:

$$
\begin{aligned}
& Y=H_{S} X+V_{r} \\
& Z=G_{S} X+V_{e}
\end{aligned}
$$

where $X$ is the input signal vector, $Y$ is the observation of the legitimate receiver, $Z$ is the observation of the eavesdropper, $H_{S}$ and $G_{S}$ are the $N_{r} \times|S|$ and $N_{e} \times|S|$ channel matrices at the legitimate receiver and the eavesdropper, respectively. $V_{r}$ and $V_{e}$ are independent and identically distributed (i.i.d.) additive 
vector Gaussian noise processes with zero means and identity covariance.

We assume that $H_{S}$ and $G_{S}$ are known at both the coalition $S$ of transmitters and the controller node. The secrecy capacity of the MIMO channel is defined as the maximum amount of reliable information transmitted from the cooperative sensor nodes to the corresponding controller node. It depends on the power constraint on the transmitted signal $X$. Let $Q_{S}=\mathrm{E}\left[X X^{\dagger}\right]$ be the Gaussian signal covariance, then the secrecy capacity of the coalition $S$ is given by [13]:

$$
\begin{gathered}
C_{S}{ }^{S}=\log \operatorname{det}\left(I_{N_{r}}+H_{S} Q_{S} H_{S}^{\dagger}\right)-\log \operatorname{det}\left(I_{N_{e}}+G_{S} Q_{S} G_{S}^{\dagger}\right) \\
=(2) \\
=\left(C_{R}^{S}-C_{E}^{S}\right)^{+}
\end{gathered}
$$

where $C_{R}^{S}$ is the capacity at the receiver, $C_{E}^{S}$ is the capacity at the eavesdropper and $a+\triangleq \max (a, 0)$.

The remaining challenge that arises in this stage is to maximize the global secrecy capacity while considering the power cost during information exchange between cooperative nodes.

\section{B. Energy consumption model}

In the resource-constrained WBAN environment, adaptive cooperation between sensor nodes according to their residual energy is required to achieve a tradeoff between the protection level provided by the virtual MIMO and the associated power consumption. The energy state of a medical device changes after each data transmission. We consider a linear discharge model with relaxation effect for a rechargeable Lithium battery. The energy depletion process is modeled as follows [14]:

$$
E_{k}(t)=E_{k}-\int_{t_{0}}^{t_{0}+t} I\left(t_{0}\right) d t
$$

where $E_{k}$ is the previous amount of energy and $I\left(t_{0}\right)$ is the instantaneous consumed energy at time $t_{0}$.

The sensor node decides to cooperate with other nodes or to remain in stand-by state on the basis of the comparison of the battery state with a fixed threshold $\xi$.

$$
\left\{\begin{array}{l}
\text { if } \frac{E_{k}(t)}{B} \geq \xi \text { Tx is On } \\
\text { if } \frac{E_{k}(t)}{B}<\xi \text { Tx is Off }
\end{array}\right.
$$

where $B$ is the battery capacity. The choice of $\xi$ depends on the required energy to transmit the sensed data. It is proved that threshold selection improves the network performance in terms of achieved capacity, however, it needs global channel knowledge for every transmission epoch.

\section{Power constraints model}

In a distributed WBAN environment, per-antenna power constraint is considered as a more realistic scenario. In fact, we incorporate the per-antenna power constraint because each antenna is equipped with its own power amplifier (PA) with a limited power budget. In the following, we first define the perantenna power constraint model. Then, we study the cost of cooperation between sensor nodes.

\section{a) Per-antenna power constraint}

In a distributed and cooperative virtual MIMO system, each transmit antenna belongs to a sensor node and cannot share power. Therefore, we assume that each sensor node has a separate transmit power budget of $\widetilde{P}_{k}(k=1 \ldots|S|)$ and it can fully cooperate with other members of the coalition $S$. The perantenna power constraint on the signal covariance matrix $Q_{S}$ is given by [15]:

$$
\left[Q_{S}\right]_{k k} \leq \tilde{P}_{k}
$$

\section{b) Cooperation power cost}

In order to form virtual MIMO coalitions and improve the secrecy capacity gains, the cooperative nodes need to exchange their data and their channel conditions. This information exchange introduces a penalty cost in terms of the transmit power. In the considered TDMA system, each coalition transmits in a slot, hence, the inter-coalition interference is eliminated. During the time slot owned by a sensor node $k \in S$, the node uses a portion of the total available power for information exchange while the remaining power $P_{k}^{S}$ is used by the coalition $S$ to transmit the node's data to the controller node. The cost of information exchange can be defined as the maximum transmitted power required by the sensor node $k \in S$ to reach the farthest sensor node in the coalition. Due to the broadcast nature of the wireless channel, once a coalition member broadcasts its information to the farthest sensor node, the other members of $S$ can also receive this information simultaneously. The transmit power required for the communication between a sensor node $k$ and the farthest node $\hat{k} \in S$ is defined as [12]:

$$
\overline{P_{k, \hat{k}}}=\frac{v_{0} \sigma^{2}}{q_{k, \widehat{k}}^{2}}
$$

where $v_{0}$ is the minimum SINR required for information exchange, $\sigma^{2}$ is the noise power and $q_{k, \hat{k}}=1 / d_{k, \hat{k}}{ }^{\mu}$ is the channel gain between nodes $k$ and $\hat{k}$ with a path loss exponent $\mu$ and a distance between nodes $k$ and $\hat{k}, d_{k, \hat{k}}$. Hence, the remaining maximum power used to transmit the data of the node $k$ during its time slot is given by:

$$
P_{k}^{S}=\left(\sum_{i \in S_{k}} P_{i}-\overline{P_{k, \hat{k}}}\right)^{+}
$$

where $P_{i}$ is the transmit power consumption of each node inside the coalition $S_{k}, \overline{P_{k, \hat{k}}}$ is the information exchange power cost given in (6).

\section{PHYSICAl LAYER SECURITY BASED ON COALITION GAME}

In this section, we formulate the cooperative transmission among sensor nodes as a coalition formation game with nontransferable utility and we study its properties. Different from canonical coalitional game, coalition formation game is considered as an efficient mechanism to model the dynamic context changes in terms of energy state and channel propagation. The players of the game are the senor nodes 
collecting sensitive and physiological data. They choose to join or break the coalition and, therefore, to cooperate or not with each other on the basis of their battery level state. The main purpose of the cooperation among the sensor nodes is to maximize their secrecy capacity in a power-constrained environment. The virtual MIMO network that transmits data to the controller node is dynamically configured at each epoch by the nodes that are part of the coalition $S$.

\section{A. Coalition game formulation}

Coalition formation game can model dynamic situations where the network structure and the cooperation cost in terms of energy consumption change frequently. In such game, the coalition brings gains to the players, however, their profits are constrained by the cooperation cost. Therefore, the main purpose of a coalition formation is to investigate the coalition structure $S$, namely: the optimal selection of cooperators, the optimal coalition size, etc. The coalition formation cost implies the negotiation or the information exchange between the communicated parties to define the coalition structure.

Definition 1: A coalition formation game is defined by the triplet $(\mathcal{N}, V, S)$ where $\mathcal{N}$ is the set of players and $V$ is a mapping such that for every coalition $S \subseteq \mathcal{N}, V(\mathrm{~S})$ is a characteristic function that determines the achieved utility by the players in $S$.

For the proposed coalition formation game, a coalition $S$ denotes the set of cooperative sensor nodes and $\varphi_{k}(S)$ is the payoff of the sensor node $k \in S$ during its time slot, we define the coalition value as follows:

$$
\begin{gathered}
V(S)=\left\{\varphi ( \mathrm { S } ) \in \mathbb { R } ^ { | S | } | \forall k \in S , \varphi _ { k } ( S ) = C _ { s } ^ { S } \text { for } \left(\left[Q_{S}\right]_{k k} \leq \tilde{P}_{k}, P_{k} S\right.\right. \\
\left.>0), \varphi_{k}(S)=-\infty \text { otherwise }\right\}
\end{gathered}
$$

where $C_{s}^{S}$ is the gain in terms of secrecy capacity for the sensor node $k \in S$ given by (2) while taking into account the total available power and the maximal transmission power of each sensor node in (7) and (5), respectively. We notice that when all the available power is spent for information exchange, the node has no advantage in cooperating and its payoff $\varphi_{k}(S)$ is set to $-\infty$. Generally, (8) assesses the benefit from cooperation in terms of secrecy gain while considering power consumption cost for information exchange. For the proposed coalitional game with non-transferable utility, we have the following properties:

Property 1: The proposed game has a non-transferable utility (NTU) whenever the sensor nodes transmit at their maximum power.

Proof: All the members of the coalition $S$ will lose communication ability, if there is a node without sufficient residual energy, and the network configuration will no longer be the same. Based on [16], it exists a unique optimal covariance matrix $Q_{S}^{*}$ that maximizes the secrecy capacity, thus, when the coalition members transmit at their maximum power the coalition value of the game $V(\mathrm{~S})$ is a singleton set, and hence, closed and convex. Consequently, the coalition value cannot arbitrary be apportioned among the members of a coalition and the proposed game has a NTU.

Property 2: For the proposed game, the grand coalition of all the nodes does not always form due to the energy constraint and power cost for information exchange.

Proof: The main purpose of the cooperation among sensor nodes is to improve the secrecy capacity while satisfying the energy efficiency requirement. However, the advantage of cooperation cannot always be achieved due to the required benefit-cost tradeoff for the coalition formation. Specifically, the cooperation cost is induced by the power consumption during information exchange. In fact, if a sensor node has not enough residual energy, it has no interest to join the coalition. In addition, if there are distant nodes that need an information power cost of $\widetilde{P}_{k}$, they have no incentive to belong to the grand coalition. In such cases, coalitions have advantage to deviate from the grand coalition and form disjoint independent coalitions. Clearly, the grand coalition is not always the optimal network structure.

Definition 2: A coalitional game $(N, V)$ with Non-Transferable Utility(NTU) is said to be super-additive if and only if:

$$
\forall S_{1}, S_{2} \subset N, V\left(S_{1} \cup S_{2}\right) \geq V\left(S_{1}\right)+V\left(S_{2}\right)
$$

Theorem 1: The proposed coalitional game for adaptive physical layer security $(N, V)$ is a non-super-additive game.

Proof: Consider two disjoint coalitions $S_{1} \subset N$ and $S_{2} \subset N$, statisfying $S=S_{1} \cup S_{2}$ and their utilities are denoted as $V\left(S_{1}\right)$ and $V\left(S_{2}\right)$, respectively. The non-super-additivity of the proposed game can be justified through two case studies as follows.

\section{- $\underline{\text { Case } 1}$}

We consider a special case in which the coalition $S_{1}$ reaches a high secrecy rate while the coalition $S_{2}$ achieves low secrecy. Such a case can occur when the players in $S_{1}$ and $S_{2}$ are nearby to the controller node and the eavesdropper, respectively. We assume that a sensor node $\mathrm{k} \in S_{2}$ has a positive coalition value $\varphi_{k}\left(S_{2}\right)$. Obviously, when $S_{1}$ and $S_{2}$ are merged, $C_{s}^{S_{1}+k}-$ $C_{s}^{S_{1}} \leq 0$, which implies that the overall secrecy capacity of the coalition is decreased. In this case, $\varphi_{k}\left(S_{1} \cup S_{2}\right)<0$, i.e. the sensor node's coalition value would be null in the merged coalition S. However, in the original coalition $S_{2}$, the sensor's coalition value is positive. Therefore, the proposed coalitional game for physical layer security is non super-additive.

\section{- $\underline{\text { Case } 2}$}

We assume that the sensor nodes of $S_{1} \cup S_{2}$ are located far enough, and thus they cannot meet the power cost constraint $P_{k}{ }^{S_{1} \cup S_{2}}>0$. In this case, $P_{k}{ }^{S_{1} \cup S_{2}}=0$ yielding $V\left(S_{1} \cup S_{2}\right)=$ $0<V\left(S_{1}\right)+V\left(S_{2}\right)$; Therefore, the proposed game is not super-additive.

The non-super-additivity implies that the coalition with more participants may not be always the optimal structure. That is explained by the fact that the benefits ensured by a coalition, 
in terms of secrecy capacity, may be restricted by the communication cost. In this context, the coalition with an extensive number of sensor nodes is not beneficial and will not be formed.

\section{B. Distributed coalition formation algorithm}

In this subsection, we design a coalition formation algorithm for the physical layer security problem. We assume that each round lasts long enough to perform the coalition formation process and the sensor nodes have sufficient processing capabilities.

Definition 3 : A collection of coalitions $S$ is defined as a set $S=\left\{S_{1}, \ldots, S_{n}\right\}$ of mutually disjoint coalitions $\mathrm{S}_{\mathrm{i}} \subset \mathcal{N}$ which does not span all players of $\mathcal{N}$. Generally, there are fundamental criteria that should be considered while implementing a coalition formation algorithm [17]. There are presented as follow:

- A right order to compare collections of coalitions;

- A set of rules to form or break coalitions;

- Suitable notions to judge the stability of the coalition.

The main purpose of the proposed game is to ensure an optimal tradeoff between security effectiveness and energy efficiency. In this context, we use the Pareto order to select the most desirable coalition. Consider two collections $S=$ $\left\{S_{1}, \ldots, S_{m}\right\}$ and $S^{\prime}=\left\{S_{1}^{\prime}, \ldots, S_{n}^{\prime}\right\}$ that have the same players. Denote $x$ and $y$ two allocations that are allotted by $S$ and $S^{\prime}$, respectively. $S$ is preferred over $S^{\prime}$ by the Pareto order $\left(S \triangleright_{\text {pareto }} S^{\prime}\right)$ if at least one player can enhance its utility in $S$ without affecting the other members, i.e., $x \geq y$ with at least one element $x_{i}$ of $x$ and $y_{i}$ of $y$, such that $x_{i}>y_{i}$. The coalitions are dynamically formed based on the following merge-and-split rules:

- Merge Rule: Merge any disjoint collections $\left\{S_{1}^{\prime}, \ldots, S_{n}^{\prime}\right\}$, if $\left\{\bigcup_{i=1}^{n} S_{i}^{\prime}\right\} \triangleright_{\text {pareto }}\left\{S_{1}^{\prime}, \ldots, S_{n}^{\prime}\right\}$, thus, small coalitions choose to merge into a larger coalition $\left\{S_{1}^{\prime}, \ldots, S_{n}^{\prime}\right\} \rightarrow\left\{\bigcup_{i=1}^{n} S_{i}^{\prime}\right\}$

- Split Rule: Split any collection $\left\{\bigcup_{i=1}^{n} S_{i}^{\prime}\right\}$, if $\left\{S_{1}^{\prime}, \ldots, S_{n}^{\prime}\right\} \triangleright_{\text {pareto }}\left\{\bigcup_{i=1}^{n} S_{i}^{\prime}\right\}$, thus, a larger coalition aims to split into disjoint collections $\left\{\bigcup_{i=1}^{n} S_{i}^{\prime}\right\} \rightarrow$ $\left\{S_{1}^{\prime}, \ldots, S_{n}^{\prime}\right\}$.

According to the above rules, the proposed physical layer security game can be solved through a distributed coalition formation algorithm, given in Algorithm 1, that is articulated in three phases. Details are described as follows:

- Neighbor discovery phase: During this phase, the proposed algorithm initializes the network on the basis of the residual energy of each node and channel state information. Each player broadcasts its information to nearby nodes and asks them if they have enough residual energy. Each node satisfying the threshold condition $\frac{E(t)}{B} \geq \xi$ sends a reply message to be a cooperator node. Thus, an initial coalition is formed and temporary cooperative nodes are selected. In the beginning, we assume that all nodes are non-cooperative. Therefore, the partition structure is initialized as $T:=\left\{S_{1}, \ldots, S_{N}\right\}$, where $S_{k}(k=1,2, \ldots N)$ includes only one node.

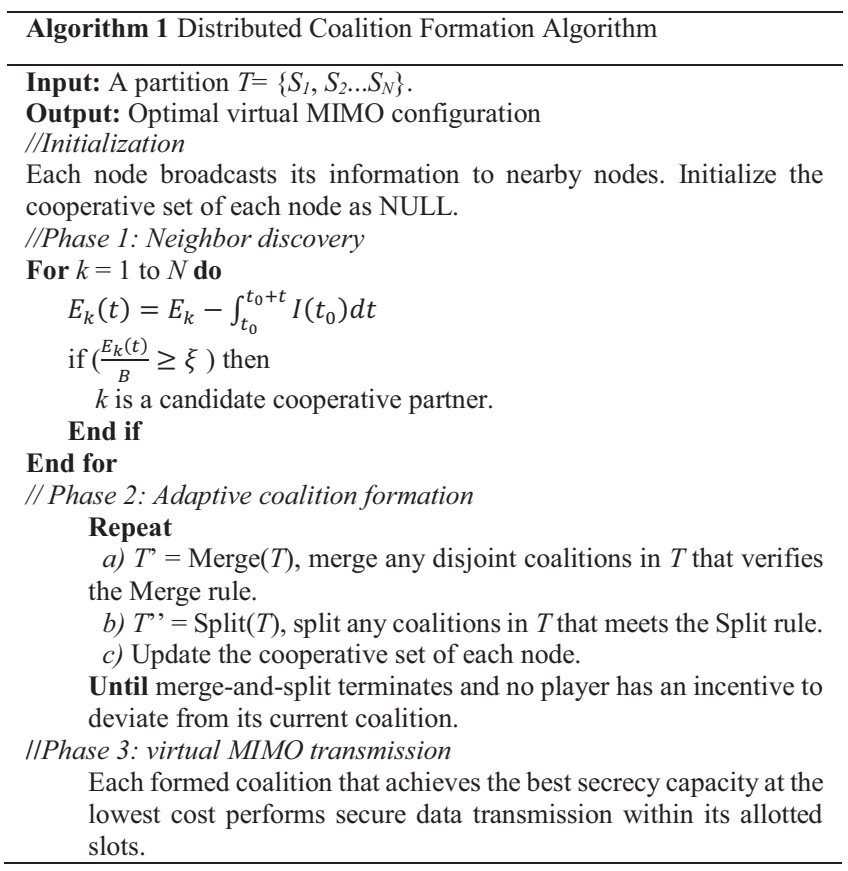

- Adaptive coalition formation phase: In this phase, the set of cooperative nodes is adjusted adaptively according to merge-and-split operations. Each individual player surveys its discovery area to select potential cooperators on the basis of the network status (energy, channel state, transmission capability, etc.). For a coalition $S_{k}$, the discovery area is determined by the distance where the power cost for information exchange does not exceed the available power of any node. Then, the Max-Pareto order is combined with successive merge-and-split operations to reach a stable coalition. The termination of merge and split iteration rules is guaranteed [17].

- Virtual MIMO transmission phase: The formed coalitions exchange their information and perform secure TDMA transmission to the controller node. Every slot is owned by a node that implements cooperative transmission. In our case, the MIMO network configuration can change epoch after epoch according to the network status in terms of channel conditions and the residual energy of nodes. In this context, each sensor node can adaptively adjust the network structure using appropriate merge-and-split operations during Phase 2. Consequently, we are speaking about a dynamically-configured virtual MIMO. Indeed, the MIMO system is virtual because it is formed by the selected cooperating single-antenna of the sensor nodes and dynamically configured because the coalition members can change in Phase 2. 


\section{Stability and complexity analysis}

Theorem 2: Every iteration of the merge-and-split rules terminates [17].

Theorem 2 proves the convergence of the proposed algorithm. The complexity of Algorithm 1 lies in the number of the merge-and-split operations. Specifically, when a given coalition will attempt to merge with other coalitions, it should verify the energy constraints. In the most complex case, each node tries to merge with all the others. In such scenario, the first node requires $N-1$ attempts for merge, the second requires $N-$ 2 attempts and so on. The total number of the required merge operations will be $\sum_{i=1}^{N} i=\frac{N(N-1)}{2}$. In practice, the merge operation needs a significantly lower number of movements because the sensor nodes can only try to merge with coalitions within their communication range that also have sufficient residual energy. The splitting decision occurs when a node $k$ wants to split and forms a coalition with other $i$ members in the coalition $S_{k}$ where $i<\left|S_{k}\right|$. We assume that $S_{k}^{\prime}=$ $\left\{C_{1}, C_{2}, \ldots, C_{\left|S_{k}\right|-1}\right\}$ is the set of all possible i-member coalitions with $i$ members. $C_{i}$ is a coalition with $i$ members for the node $k$ that is composed of the node $k$ and $i-1$ other members from $S_{k}$. The total number of split moves for each node $k$ is $\left|S_{k}^{\prime}\right|$. The most complex splitting case occurs when the network forms the grand coalition. However, this complexity is reduced by the fact that the size of a coalition is typically small due to the cooperation cost and energy constraints. In this context, the split operations will be limited to small partition sets. Therefore, we will seldom encounter the grand coalition. Indeed, the size of the most formed coalitions will be much smaller than the grand coalition.

Definition 4: A partition structure $S=\left\{S_{1}, \ldots, S_{k}, \ldots, S_{N}\right\}$ is Nash-stable if $\forall k \in \mathcal{N}, S_{k} \geqslant S_{k^{\prime}} \cup\{k\}$ for all $S_{k^{\prime}} \in S \backslash S_{k} \cup$ $\{\varnothing\}$.

A partition structure is stable in the sense that no player $k$ has incentive to unilaterally change its current coalition $S_{k}$ to another coalition $S_{k}$. In addition, according to Definition 4, a stable partition implies that no player $k$ in current partition structure can benefit from moving to another coalition while making other players believe that their utilities would not be worse.

Property 3: The proposed algorithm of the physical layer security coalitional game converges to a Nash-stable coalitional structure $S^{*}$.

Proof: The stability of coalitions can be proved using the merge and split rules. Based on the definition of Pareto order, any sensor node $k \in \mathcal{N}$ can join any coalition only if none of that coalition members could be adversely affected. Specifically, for an arbitrary coalitional structure $S$, if there is any node $k$ that chooses to join another coalition, i.e. $\left\{\left\{S_{k}-k\right\},\left\{S_{k^{\prime}} \cup\right.\right.$ $\left.k\},\left\{S_{i}\right\}_{i \neq k, k^{\prime}}\right\} \geqslant\left\{S_{k}, S_{k^{\prime}},\left\{S_{i}\right\}_{i \neq k, k^{\prime}}\right\}$, then, the current structure $S$ is not stable. Consequently, node $k$ will leave $S_{k}$ for $S_{k}$, via an iterative merge-and-split moves. When each node gains benefit from its current situation, the iterative merge-and-split operations will stop. Indeed, in this case, no one has an incentive to change its current coalition. Therefore, a Nashstable coalitional structure $S^{*}$ is reached.

\section{PERFormance EVAluation}

In this section, we evaluate the performance of the gamebased adaptive physical layer security approach. Our main purpose is to find the most appropriate virtual MIMO configuration that ensures an optimal tradeoff between the secrecy performance and energy consumption in WBAN. For simulation, we consider a WBAN consisting of twenty on-body sensor nodes. The WBAN has one controller node with multiple antenna, and several eavesdroppers are present in the vicinity. Furthermore, let $h_{i, k}$ be the quasi-static fading channel modeled by $h_{i, k}=e^{j \varphi_{i, k}} \sqrt{\kappa / d_{i, k}^{\mu}}$ where $\mu$ the propagation loss exponent, $\kappa$ the path loss constant, $d_{i, k}$ and $\varphi_{i, k}$ the Euclidean distance and the phase offset between nodes $i$ and $k$, respectively. Particularly, the propagation loss $\mu$ is set to 3 and the path loss constant $\kappa$ is set to 1 during the simulation. Moreover, the power constraint of each sensor node is set to 10 $\mathrm{mW}$, and the noise variance is set to $-90 \mathrm{dBm}$.

At first, we evaluate the secrecy performance of the cooperative transmission among sensor nodes. Figure 2 shows the secrecy capacity versus $\mathrm{SNR}(\mathrm{dB})$ for different virtual MIMO configurations. The channel performance in terms of secrecy capacity consistently increases according to spatial diversity. As we can see that the $4 \times 4$ virtual MIMO configuration (4 sensor nodes- 4 antennas in the receiver) is characterized by the highest secrecy capacity when compared to the $2 \times 2$ lower order system. Therefore, cooperative transmission among medical devices improves their secrecy capacity.

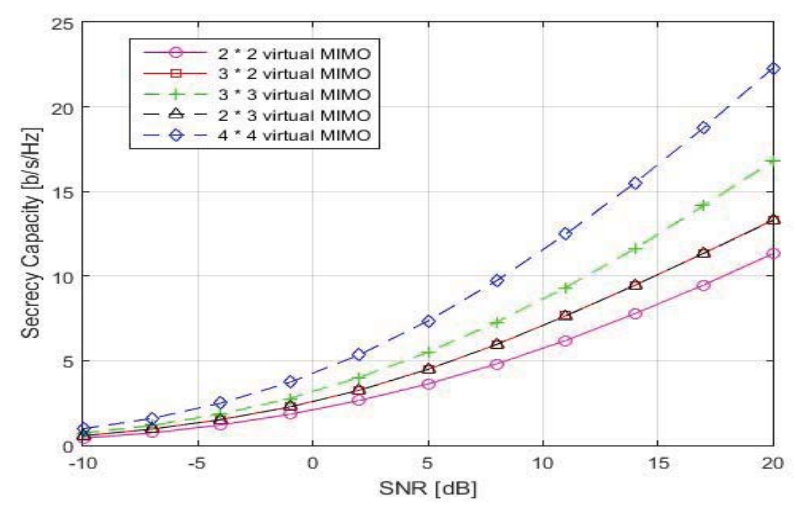

Figure 2. The impact of virtual MIMO on secrecy capacity

Next, we study the impact of the proposed adaptive virtual MIMO configuration on the system performance in terms of energy consumption for a $4 \times 4$ virtual MIMO configuration. As shown in Figure 3, the proposed virtual MIMO configuration approach outperforms the non-cooperative transmission in terms of network lifetime which is defined as the residual energies of sensor nodes after each transmission. In fact, noncooperative transmission leads to a lower performance where the energy is depleted after around 800 time slots. However, the cooperative MIMO extends the network lifetime where the 
energy is depleted after 1600 time slots. We note that the performance of the cooperative transmission is enhanced because we introduced the residual energy threshold in the cooperative decision.

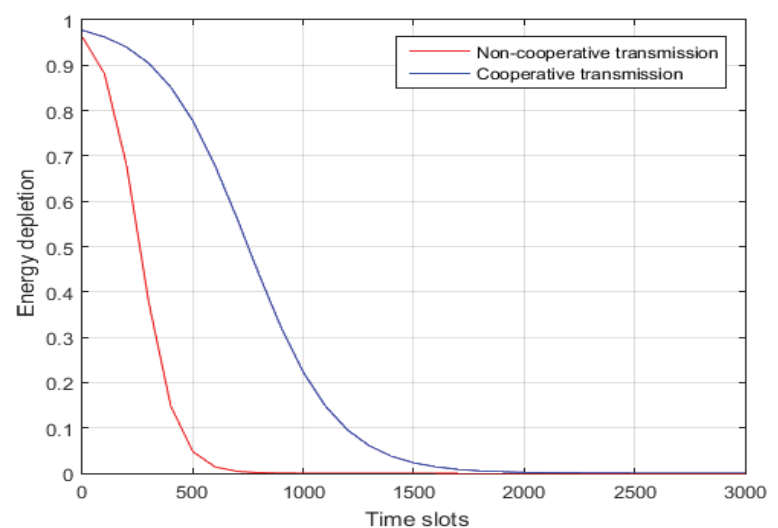

Figure 3. Performance evaluation in terms of network lifetime

Finally, Figure 4 illustrates the evolution of the utility per device, as a function of the number of the medical devices. For the cooperative transmission based on coalition formation game, the average individual utility increases with the number of sensor nodes. This is explained by the fact that when the number of sensor nodes increases, the probability of finding proper candidate partners to form coalitions increases. However, the non-cooperative transmission admits low secrecy performance. Thus, cooperation based on virtual MIMO configuration performs a clear performance improvement in terms of average utility per device.

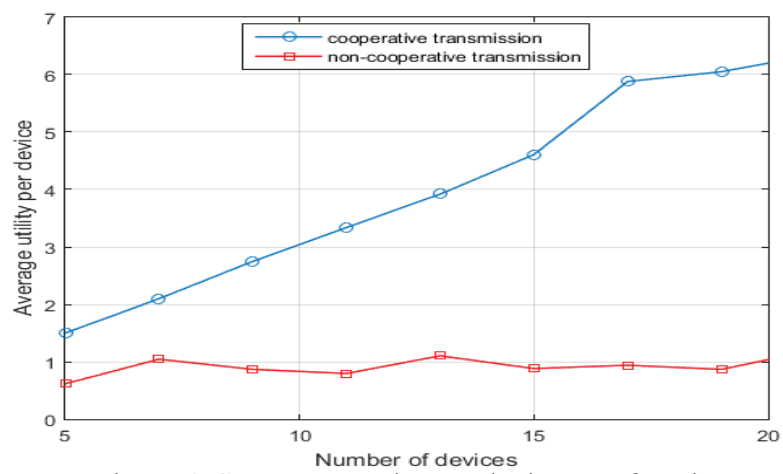

Figure 4. Secrecy capacity per device as a function of the number of sensor nodes

\section{V.CONCLUSION}

In this paper, we addressed the adaptive physical layer security in IoT for e-Health application in order to ensure a tradeoff between the security effectiveness (secrecy capacity) and the energy efficiency. In our model, a dynamic virtual MIMO configuration approach was performed while considering the residual energy of the medical devices. In this context, we proposed a coalition formation game to select the most suitable cooperative partners. Then, we evaluated the performance of the virtual MIMO configuration in terms of secrecy capacity and energy consumption. We demonstrated the effectiveness and efficiency of the proposed approach as compared to non-cooperative transmission.

\section{REFERENCES}

[1] M. Bloch, J. Barros, M. Rodrigues, and S. McLaughlin, "Wireless information-theoretic security," IEEE Trans. Inf. Theory, vol. 54, no. 6, pp. 2515-2534, June 2008.

[2] N. Yang et al., "Safeguarding 5G wireless communication networks using physical layer security," IEEE Commun. Mag., vol. 53, no. 4, pp. 20-27, Apr. 2015.

[3] A. Mukherjee, "Physical-layer security in the Internet of Things: Sensing and communication confidentiality under resource constraints," Proc. IEEE, vol. 103, no. 10, pp. 1747-1761, Oct. 2015.

[4] L. Xiao, T. Chen, G. Han, Y. Li, W. Zhuang, and L. Sun, "Channel-based authentication game in MIMO systems," in Proc. IEEE Global Commun. Conf., Washington, DC, USA, Dec. 2016, pp. 1-6.

[5] N. Yang, M. Elkashlan, T. Q. Duong, J. Yuan, and R. Malaney, "Optimal transmission with artificial noise in MISOME wiretap channels," IEEE Trans. Veh. Technol., vol. 65, no. 4, pp. 2170-2181, Apr. 2016.

[6] N. Yang, P. L. Yeoh, M. Elkashlan, R. Schober, and I. B. Collings, "Transmit antenna selection for security enhancement in MIMO wiretap channels," IEEE Trans. Commun., vol. 61, no. 1, pp. 144-154, Jan. 2013.

[7] N. Yang, H. A. Suraweera, I. B. Collings, and C. Yuen, "Physical layer security of TAS/MRC with antenna correlation," IEEE Trans. Inf. Forensics Security, vol. 8, no. 1, pp. 254-259, Jan. 2013.

[8] N. Zhang, N. Cheng, N. Lu, X. Zhang, J. Mark, and X. Shen, "Partner selection and incentive mechanism for physical layer security," IEEE Trans. Wirel. Commun., vol. 14, no. 8, pp. 4265-4276, Aug. 2015.

[9] Y. Zou, X. Wang, and W. Shen, "Optimal relay selection for physicallayer security in cooperative wireless networks," IEEE J. Sel. Areas Commun., vol. 31, no. 10, pp. 2099-2111, Oct. 2013.

[10] H. Moosavi and F. M. Bui, "Delay-aware optimization of physical layer security in multi-hop wireless body area networks," IEEE Trans. Inf. Forensics Security, vol. 11, no. 9, pp. 1928-1939, Sep. 2016.

[11] M. Augier and D. Teece, The Palgrave Encyclopedia of Strategic Management. Springer, 2013.

[12] W. Saad, Z. Han, M. Debbah, A. Hjorungnes and T. Basar, "Coalitional game theory for communication networks," in IEEE Signal Processing Magazine, vol. 26, no. 5, pp. 77-97, September 2009.

[13] F. Oggier and B. Hassibi, "The Secrecy Capacity of the MIMO Wiretap Channel," in IEEE Transactions on Information Theory, vol. 57, no. 8, pp. 4961-4972, Aug. 2011.

[14] Mohamed Hamdi, Habtamu Abie, "Game-Based Adaptive Security in the Internet of Things for eHealth", IEEE International Conference on Communications, June.2014

[15] M. Vu, "MIMO Capacity with Per-Antenna Power Constraint," 2011 IEEE Global Telecommunications Conference - GLOBECOM 2011, Kathmandu, 2011, pp. 1-5.

[16] S. Yerramalli, R. Jain and U. Mitra, "Coalition games for transmitter cooperation in wireless networks," 2011 IEEE International Symposium on Information Theory Proceedings, St. Petersburg, 2011, pp. 2706-2710.

[17] L. Chen, W. Wang and C. Zhang, "Coalition Formation for Interference Management in Visible Light Communication Networks," in IEEE Transactions on Vehicular Technology, vol. 66, no. 8, pp. 7278-7285, Aug. 2017. 